\title{
Population synthesis as a probe of neutron star thermal evolution
}

\author{
S. Popov ${ }^{1}$, H. Grigorian ${ }^{2,3}$, R. Turolla ${ }^{4}$, and D. Blaschke $e^{5,6, \star}$ \\ 1 Sternberg Astronomical Institute, Universitetski pr. 13, 119992 Moscow, Russia \\ 2 Department of Physics, Yerevan State University, Alex Manoogian Str. 1, 375049 Yerevan, Armenia \\ 3 Institut für Physik, Universität Rostock, 18051 Rostock, Germany \\ ${ }^{4}$ Università di Padova, Dipartimento di Fisica, via Marzolo 8, 35131 Padova, Italy \\ 5 Fakultät für Physik, Universität Bielefeld, 33615 Bielefeld, Germany \\ e-mail: blaschke@theory.gsi.de \\ ${ }^{6}$ Bogoliubov Laboratory of Theoretical Physics, Joint Institute for Nuclear Research, 141980 Dubna, Russia
}

Received 23 November 2004 / Accepted 18 October 2005

\section{ABSTRACT}

The study of thermal emission from isolated, cooling neutron stars plays a key role in probing the physical conditions of both the star crust and the core. The comparison of theoretical models for the star thermal evolution with the surface temperature derived from X-ray observations of sources of different age is one of the main tools to investigate the properties of the interior and constrain the equation of state. Here we propose to use population synthesis studies as an independent approach to test the physics governing the star cooling. Theoretical Log $N-\log S$ distributions depend on the assumed neutron star thermal evolution. We have computed distributions for several different cooling scenarios and found that comparison with the observed $\log N-\log S$ of isolated neutron stars is effective in discriminating among cooling models. Among the eleven cooling models considered in this paper, all of which may reproduce the observed temperature vs. age diagram, only at most three can explain the $\log N-\log S$ distribution of close-by cooling neutron stars. The $\log N-\log S$ test, being a "global" one and despite some limitations, appears indeed capable to ideally complement the standard temperature vs. age test used up to now.

Key words. stars: evolution - stars: neutron - X-rays: stars

\section{Introduction}

The determination of the equation of state (EOS) at or above nuclear densities is a long sought goal in high energy astrophysics. In this respect direct observations of neutron stars (NSs) are bound to provide invaluable insight into several key issues of fundamental physics, like quantum chromodynamics/electrodynamics, superfluidity and superconductivity, that could not be otherwise tested under laboratory conditions. An ideal way to place tight constraints on the EOS is the simultaneous mass and radius measurement of NSs (see e.g. Lattimer $\&$ Prakash 2001). Precise mass determinations have been obtained for NSs in binaries, especially in radio pulsar systems (e.g. Thorsett \& Chakrabarty 1999). Simultaneous mass and radius measurements are presently available for a few X-ray binaries, although masses derived from gravitational redshift of spectral lines are still uncertain in many cases (e.g. Cottam et al. 2002). Mass estimates can be obtained also from quasiperiodic oscillation measurements (Bulik et al. 2000), but results based on this, as well as other approaches, are still quite model dependent. The study of glitches observed in radio pulsars, and recently in anomalous X-ray pulsars (AXPs) as well

\footnotetext{
* Present address: GSI, 64291 Darmstadt, Germany.
}

(e.g. Horvath 2004; Dall'Osso et al. 2003), promise further insight into the understanding of the internal structure of NSs, as well as future observations of neutrino and gravitational wave emission from neutron star sources.

For the time being, however, X-ray observations of NSs are, and will still be for some time, central in fostering our understanding of the star interior. Isolated neutron stars which emit at $\mathrm{X}$-ray energies as they cool are particularly promising in this respect. Their thermal radiation, in fact, directly comes from the star surface, carrying information on the physical conditions of the emitting matter, in particular on the star surface temperature. Thermal X-ray emission has been detected from about 20 isolated NSs so far, including normal radio pulsars, central compact objects in supernova remnants (CCOs in SNRs), radioquiet NSs, AXPs and soft $\gamma$-repeaters (see e.g. Pavlov \& Zavlin 2003; Kaspi et al. 2004; Haberl 2004 for reviews).

Significant progress in the understanding of NS thermal evolution has been made in recent years and cooling curves have been computed by several groups (see e.g. Kaminker et al. 2002; Tsuruta et al. 2002; Page et al. 2004; Blaschke et al. 2004, and references therein). The present work is based on NS cooling calculations performed in the Nuclear medium cooling scenario (Blaschke et al. 2004), hereafter BGV, which 
differs from the other above mentioned approaches by a consistent inclusion of medium effects. Processes of internal heating (Tsuruta et al. 2002) are not included. Since the cooling history crucially depends on the assumed physical conditions inside the star, comparison with observations may rule out some models in favor of others. In order to exemplify this we will vary assumptions on the nuclear pairing gaps, the relation between crust and surface temperature as well as presence or absence of pion condensation. A customary way of testing predictions of cooling calculations is to construct a temperature vs. age (T-t for short) plot for the largest sample of sources. Despite its wide application and undisputed usefulness, this test has a number of limitations. In this paper we suggest to use the $\log N-\log S$ distribution of close-by NSs as an additional probe for NS cooling models. The idea is based on the comparison of present observational data with NS population synthesis calculations in which cooling curves are one of the ingredients. Our approach extends the actual calculation of observational properties of the population of close-by young NSs that has already been developed by Popov et al. (2003, 2005) (hereafter Papers I and II) by including nuclear medium effects in the cooling code and by investigating the contribution of massive progenitors in the Gould Belt to the local NS population in more details.

The paper is organized as follows. In Sect. 2 we discuss the advantages and limitations of the two methods in extracting information from observational data. Section 3 presents our population synthesis model in more detail, especially concerning the choice of the set of cooling curves which will be used in our calculation. We present our results and discuss them in Sect. 4. Section 5 contains our conclusions.

\section{The two tests}

In this section we briefly discuss and compare the capabilities of the conventional T-t test and of the $\log N-\log S$ test we propose here. Our main conclusion is that the two approaches should be used together as their advantages and disadvantages are mostly complementary to each other especially in the case of available samples of observed objects.

\subsection{The T-t test}

Obviously the T-t test is the most natural one when it comes to compare results of thermal evolution calculations with observations. An important advantage of this test is that there are no additional theoretical uncertainties except those ones connected with the cooling model: theoretical cooling curves do not depend on unknown (or poorly known) astrophysical parameters but only on the input physics of the star interior (see e.g. for a discussion of cooling processes and references to earlier papers Yakovlev et al. 1999 and Blaschke et al. 2004). Still, there are some well known drawbacks in this method.

For sources associated with radiopulsars or CCOs, the star age is usually estimated from the spindown age $P / \dot{P}$, or inferred from the age of the supernova remnant. To which extent these determinations are indeed representative of the neutron star age is still uncertain. The situation is even worse for ROSAT isolated neutron stars which are not associated with a SNR and are with no exception radio-silent. Current age estimates for the two brightest objects in this class (RX J1856.5-3754 and RX J0720.4-3125), based on dynamical considerations, should be regarded only as educated guesses.

The T-t test is not very sensitive to objects with ages $\gtrsim 10^{5} \mathrm{yr}$. There are two reasons for this. In the first place, there are only few sources older than this value to which the test can be applied. Then, cooling curves sharply go down at the photon cooling stage. Shifts between different cooling curves are comparable with data error bars. So, it is difficult to discriminate between models which differ mainly in this respect.

Cooling calculations provide the star temperature at the core-crust boundary and the actual surface temperature is then obtained applying a bridging formula (e.g. Tsuruta 1979; Yakovlev et al. 2004). Detailed modeling of heat transport in the highly magnetized envelope indicates that the surface temperature may be influenced by several effects, among which the magnetic field distribution inside the star is much relevant (Geppert et al. 2004). In addition, one should bear in mind that the temperature is derived from a spectral fit to the data and, as such, depends on the assumed emission model for the surface. Different models (blackbody, H/heavy elements atmosphere with/without magnetic field, solid surface) give values which can differ by a factor of a few.

Recently, it has been suggested that the Temperature-Age test should be sharpened by the introduction of a brightness constraint (BC), which demands that cooling curves for objects within a mass interval anticipated as typical should not appear significantly brighter than the brightest of already observed objects for a given age (Grigorian 2005), hereafter (G05). It has been shown in this work that some of the cooling models discussed in BGV, which would violate the BC become acceptable when the crustal properties are modified. These modifications do not affect old stars with ages $\gtrsim 10^{5}$ yr for which the $\log N-\log S$ test is relevant. Due to our lack of knowledge of the mass distribution and atmospheric properties of cooling compact stars, we want to be cautious in applying the strong BC. Therefore we refer in this paper to results of the T-t test with and without BC.

Finally, the data sample is non-uniform: there are sources of different types, and, more important, the sample is nor flux, neither volume limited, and is a strongly selected one, containing objects for which age and temperature estimates are available ${ }^{1}$. Clearly these sources can be hardly claimed to be representative of any real population of NSs.

\subsection{The $\log N-\log S$ test}

The $\log N-\log S$ distribution is a widely used tool in many branches of astronomy. For isolated NSs such an approach has been already used by Neuhäuser \& Trümper (1999) and Popov et al. (2000) to probe the origin of isolated NSs in the solar proximity. The main conclusion of these investigations is that

\footnotetext{
1 Note that there are some young cooling NSs for which age is not known, in particular those which are not observed as active radiopulsars. These sources can not be included in the sample of the Temperature-Age test.
} 
the observed $\log N-\log S$ cannot be easily explained assuming that the local population of NSs originated only in the Galactic disc. As shown in Papers I and II, accounting for massive progenitors in the Gould Belt reconciles theoretical predictions with the data.

One immediate advantage of the $\log N-\log S$ test is that, at variance with the T-t test, no degree of arbitrariness is introduced when observational data are analyzed: both the fluxes and (of course) the number of sources are well measured. In addition, this approach is a "global" one. In our scenario it would not be possible to explain some particular sources by invoking slight changes in the cooling physics. Once the parameters of the model other than those related to the cooling process are fixed (see Sect. 3), a particular cooling curve either fits the population as a whole or not. Furthermore, the $\log N-\log S$ sample is a uniform one, i.e. objects are flux (and probably volume) limited, and no strong selection criteria are introduced.

For the $\log N-\log S$ test the only necessary observational piece of information is the ROS AT count rate. The method can be applied to objects with unknown ages. This makes possible to include, for example, all the ROSAT X-ray dim NSs, and 3EG J1835+5918 (the Geminga twin) into the testing sample. The $\log N-\log S$ test is mostly sensitive to NSs older than $\sim 10^{5} \mathrm{yr}$. Older sources dominate in number, and in the solar proximity there are about a dozen of them in comparison to very few with $t \lesssim 10^{5}$ years $^{2}$.

Nevertheless there are significant limitations too. One source of uncertainties lies in our incomplete knowledge of some important ingredients of the population synthesis model. These are discussed in more detail in the next section and concern the spatial distribution of the NS progenitors, the NS mass and velocity spectrum, and their emission properties. However, all these issues can be, to some extent, addressed by considering different cases which are believed to cover the entire range of acceptable scenarios. In addition, there could be unknown correlations among some of the quantities we use to parametrize our model, so that they should not be treated as independent ones. Examples of such possible correlations are those between the star kick and the internal structure (because of quark deconfinement, see Bombaci \& Popov 2004), and between the star mass and the magnetic field (because of fallback, see Popov et al. 2002; Heger et al. 2005). A more severe problem arises in connection with the low statistics of the sample, since there are only about 20 thermally emitting NSs known to date. This implies that the bright end to the $\log N-\log S$ comprises very few objects so that it is difficult to account for statistical fluctuations. We do not know much either about the properties of very faint sources, i.e. the dim end of the $\log N-\log S$ distribution.

\footnotetext{
${ }^{2}$ Of course, the $\log N-\log S$ test can be sensitive to young objects if another uniform sample is used. For example, it is very important to make a population synthesis of sources in $\mathrm{SN}$ remnants. Then, as we mentioned above, differences between cooling curves for large ages are tiny. However, in the $\log N-\log S$ approach we, in some sense, integrate these small differences along the curves, so their impact on the final $\log N-\log S$ distribution is significant.
}

\section{The population synthesis model}

The main physical ingredients which enter our population synthesis model are:

- the initial NS spatial distribution;

- the kick velocity distribution;

- the NS mass spectrum;

- the cooling curves;

- the surface emission;

- the interstellar absorption.

The calculation of the NS spatial evolution as they move in the Galactic gravitational potential follows that presented in Papers I and II . The same treatment of the interstellar absorption is retained and the kick distribution is that proposed by Arzoumanian et al. (2002). We do not account for atmospheric reprocessing of thermal radiation, and assume that the emitted spectrum is a pure blackbody. Although this is clearly an oversimplification, it is a reasonable starting assumption and will serve for our, mainly illustrative, purposes. A more detailed description of surface emission may be easily accommodated in our model later on. For the time being, we perform our calculations for nine different sets of cooling curves among those discussed in hereafter BGV, to which we refer for all details. This issue, together with the initial spatial distribution of NSs and their mass spectrum, is further discussed below.

\subsection{The initial NS spatial distribution}

Following the results of previous investigations (Paper I), we take as an established fact that the population of nearby NSs is genetically related to the Gould Belt. The contribution of the Belt dominated the production of compact remnants in the solar proximity over the past $\sim 30$ Myr (see Pöppel 1997 for a detailed description of the Belt structure). About two thirds of massive stars in the $\sim 600 \mathrm{pc}$ around the Sun belong to the Belt (Torra et al. 2000). Since they are bright objects, it is possible to track their positions exactly for example by using HIPPARCOS data. However, here we use a simplified distribution of the progenitor stars, as discussed in Papers I and II. NSs are born in the Belt, for which a simple ring-shaped structure is assumed, and in the Galactic disc. The outer belt radius is a model parameter and two values, $300 \mathrm{pc}$ and $500 \mathrm{pc}$, were actually used, the second being an upper limit. The supernova rate was taken from Grenier (2000), and appears in agreement with the historical rate estimated by Tammann et al. (1994).

\subsection{The NS mass spectrum}

Since cooling curves are strongly dependent on the star mass, the mass spectrum is one of the most important ingredients and, unfortunately, one of the less known. We cannot rely on the mass measurements in binary radio pulsars (e.g. Thorsett \& Chakrabarty 1999) because they refer to a "twice selected" population (i.e. selection effects due to evolution in binary can be important together with possible conditions which are necessary for a radio pulsar formation). Probably not all NSs go 


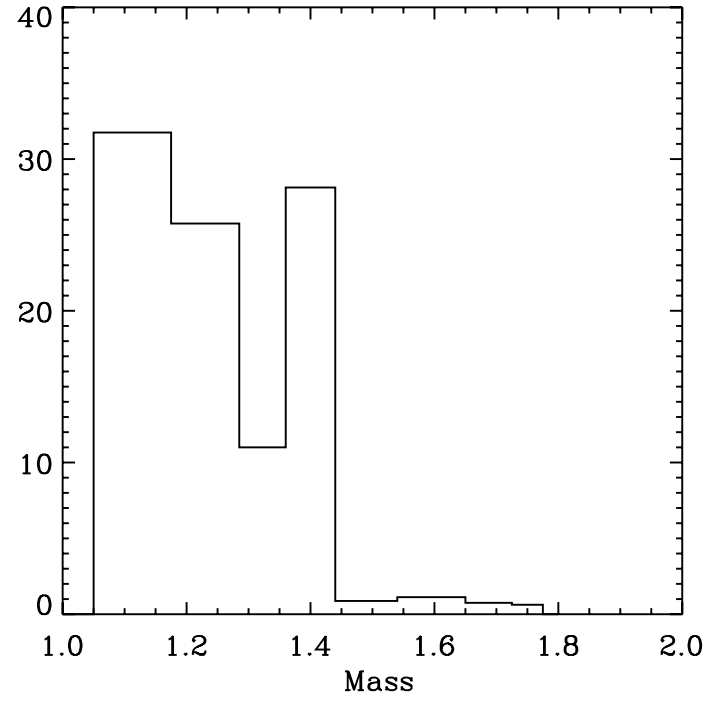

Fig. 1. The adopted mass spectrum, binned over eight intervals of different widths.

through the active radio pulsar stage (e.g. Gotthelf \& Vasisht 2000), and the properties of NSs in binaries may be different from those of isolated objects (e.g. Podsiadlowski et al. 2003). Note that the local NS mass spectrum can be different from the global NS mass spectrum in the Galaxy. Even stronger deviations can be expected between the mass spectra of local NSs and of those sources usually used for the T-t plot.

As the population of NSs in $\sim 1 \mathrm{kpc}$ around the Sun may be slightly different from the average galactic population, we estimated the mass spectrum for these objects directly (see Paper II for more details). The basic idea is to use HIPPARCOS data on massive stars around the Sun in conjunction with the calculations by Woosley et al. (2002). Knowing the mass distribution of progenitors through their spectral classes, we use a fit to a plot from Woosley et al. (2002) in order to obtain the NS mass from the mass of the progenitor.

We use eight mass bins centered at $M / M_{\odot}=1.1,1.25,1.32$, $1.4,1.48,1.6,1.7,1.76$. The adopted mass spectrum is shown in Fig. 1. The lower limit for the NS mass is still an open question. Timmes et al. (1996) suggested that there are no NSs with $M \lesssim 1.27 M_{\odot}$, although their conclusion is not definite (see also Woosley et al. 2002). For this reason we decide to use also a truncated mass spectrum, in which the first bin is suppressed and all objects originally contained there are added to the second one. Each bin corresponds to one of the calculated cooling curves. According to the mass spectrum, each curve has a statistical weight of $31.75 \%, 25.75 \%, 11 \%, 28.125 \%, 0.875 \%$, $1.125 \%, 0.75 \%$, and $0.625 \%$. For the truncated one the weights of the first two bins are replaced by 0.0 and $57.5 \%$ respectively. We note that sampling also relatively low masses is important since low-mass NSs seem to be required to interpret data on the T-t plot (BGV).

\subsection{Cooling curves}

In their recent paper Blaschke et al. (2004) presented sixteen sets of cooling curves. Each set contains models for several values of the star mass while different sets refer to different assumptions on heat transport in the crust and on the physical processes in the NS interior. Five of these models are unable to reproduce the observed temperature-age plot and will be considered no further. From the remaining eleven sets, all of which give results not in contradiction with observations (see, however, the discussion in Sect. 4), we select nine representative models for our population synthesis calculations (models I-IX in Table 1). We add two models (X and XI) from the recent analysis in G05 which correspond to models III and VII, respectively, when calculated with different crustal properties. All of them have superfluid nuclear matter and medium modifications of the neutrino processes. They differ in the assumptions about the superfluid gaps, the presence/absence of a pion condensate and the properties of the neutron star crust. The latter governs the relationship between the temperature of outermost core layer $\left(T_{\text {in }}\right)$ to that of the star surface $\left(T_{\mathrm{s}}\right)$. The main characteristics of the selected models are summarized in Table 1. All of the eleven models satisfy the temperature-age test according to BGV, whereas only six of them fulfill the additional brightness constraint introduced in G05. The last column anticipates the results discussed in the following section and shows if the model complies with the $\log N-\log S$ test.

\section{Results and discussion}

In each run we calculate 5000 individual tracks for the spatial evolution of a single star with a time step of $10^{4} \mathrm{yr}$. Each track is applied to all eight (or seven for the truncated spectrum) masses, and the thermal evolution is followed with the correspondig cooling curve. Results are then collected according to the statistical weight of each mass bin.

Results are summarized in Table 1 and Figs. 2-7, which refer to a selected sub-sample of the cooling curve sets listed in that table. In the left panels of Figs. 2-7, the corresponding cooling curves for the various masses are shown. Results are plotted for ages $>10^{4} \mathrm{yr}$ and temperatures above $10^{5} \mathrm{~K}$. The right panels illustrate the $\log N-\log S$ distributions computed for the same sets of cooling curves. All models have been calculated using both the full mass spectrum and the truncated one, although results for the latter are not shown in all cases (see the following discussion). Two values of the outer radius of the Gould Belt have been used to test the dependence of our calculation on the assumed geometry, $R_{\text {belt }}=300 \mathrm{pc}$ and $500 \mathrm{pc}$.

Theoretical distributions are superimposed to the observed $\log N-\log S$ for isolated NSs. The data points are derived from the sample of thirteen sources listed in Paper I. Error bars correspond to poissonian statistics and are plotted just to illustrate the statistical significance of the points. We note, however, that there can be more unidentified sources, especially at fluxes below $0.1 \mathrm{cts} \mathrm{s}^{-1}$ (see Rutledge et al. 2003 for a recent discussion). In this respect, the fact that the two points at the lowest fluxes lie below the general trend of the observed $\log N-\log S$ is not surprising. In addition, the observational upper limit derived by Rutledge et al. (2003) on the number of fainter sources is also shown (marked as BSC in the figures). Bounds on on the total number of sources with flux $>0.2 \mathrm{cts} \mathrm{s}^{-1}$ have been also 
Table 1. Properties of the selected cooling curves: A - gaps from Takatsuka \& Tamagaki (2004), ${ }^{3} \mathrm{P}_{2}$ neutron gap suppressed by 0.1 ; B - gaps from Yakovlev et al. (2004), ${ }^{3} \mathrm{P}_{2}$ neutron gap suppressed by $0.1 ; \mathrm{B}^{\prime}-$ same as for $\mathrm{B}$ and ${ }^{1} \mathrm{P}_{0}$ proton gap suppressed by 0.5 ; $\mathrm{B}^{\prime \prime}-$ same as for $\mathrm{B}$, ${ }^{1} \mathrm{P}_{0}$ proton gap suppressed by 0.2 and ${ }^{1} \mathrm{P}_{0}$ neutron gap suppressed by $0.5 ; \mathrm{C}-T_{\mathrm{s}}-T_{\text {in }}$ relation fit from BGV; D $-T_{\mathrm{s}}-T_{\text {in }}$ relation by Tsuruta (1979); $\mathrm{E}-T_{\mathrm{s}}-T_{\text {in }}$ relation from Yakovlev et al. (2004) and $\eta=4 \times 10^{-16}$. The last three entries indicate whether the model complies or not with the tests: temperature-age without (wo) or with (w) the additional brightness constraint and $\log N-\log S$ (see text and also BGV and G05 for more details).

\begin{tabular}{cccccccc}
\hline \hline Model & Reference & $\pi$ Cond & Gaps & Crust & \multicolumn{2}{c}{ T-t } & Log $N-$ Log $S$ \\
& & & & & wo BC & w BC & \\
\hline I & BGV, Fig. 21 & Yes & A & C & Yes & Yes & Yes \\
II & BGV, Fig. 13 & No & B & D & Yes & No & No \\
III & BGV, Fig. 15 & Yes & B & C & Yes & No & No \\
IV & BGV, Fig. 12 & No & B & C & Yes & No & No \\
V & BGV, Fig. 16 & Yes & B & D & Yes & No & No \\
VI & BGV, Fig. 14 & No & B & E & Yes & Yes & No \\
VII & BGV, Fig. 18 & Yes & B' & C & Yes & No & No \\
VIII & BGV, Fig. 19 & Yes & B" & C & Yes & Yes & Yes \\
IX & BGV, Fig. 20 & No & A & C & Yes & Yes & Yes \\
X & G05, Fig. 2 & Yes & B & E & Yes & Yes & No \\
XI & G05, Fig. 2 & Yes & B' & E & Yes & Yes & No \\
\hline
\end{tabular}
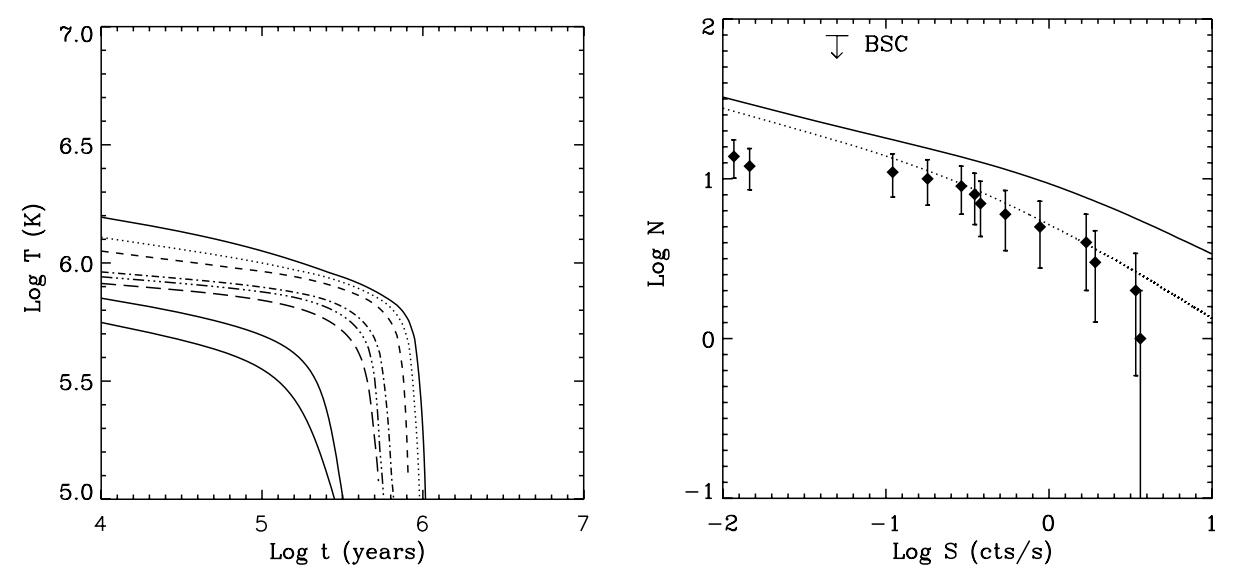

Fig. 2. Model I. Left: cooling curves for (from top to bottom) 1.1, 1.25, 1.32, 1.4, 1.48, 1.6, 1.7, 1.76 $M_{\odot}$. Right: the corresponding Log $N-\log S$ distribution for $R_{\mathrm{belt}}=300 \mathrm{pc}$ and non-truncated mass spectrum (full line) and $500 \mathrm{pc}$ and truncated mass spectrum (dotted line). See text for details.
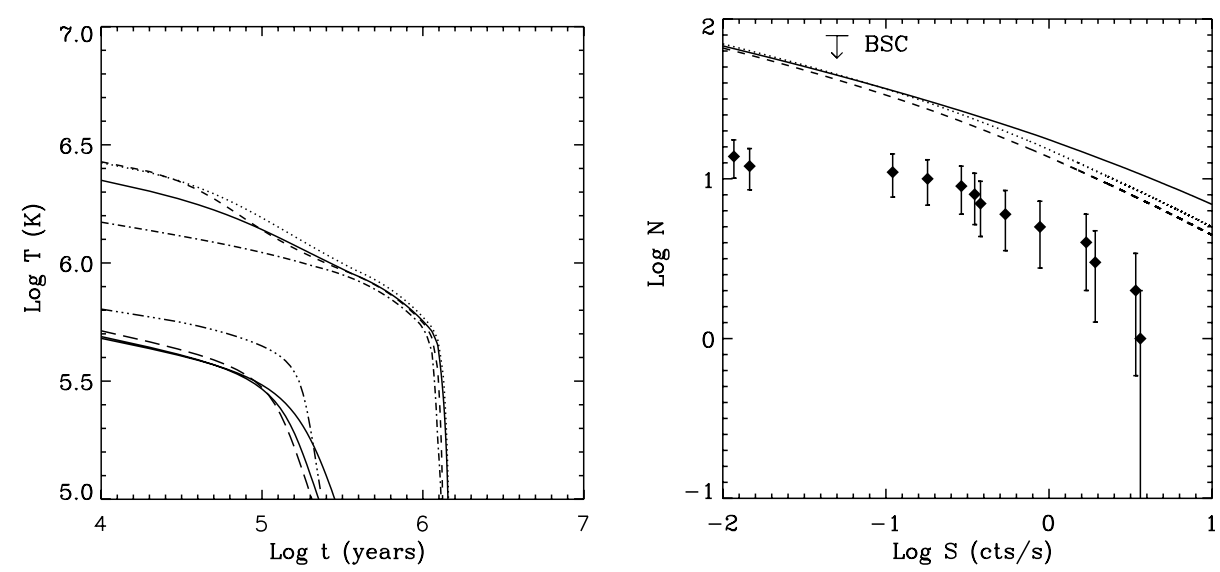

Fig. 3. Same as in Fig. 2 for Model III. The dashed line refers to a calculation in which the full (non-truncated) mass spectrum was used and $R_{\text {belt }}$ was assumed to be $500 \mathrm{pc}$. Model X should produce nearly the same $\log N-\log S$ distribution as it differs only in the type of the crust. 

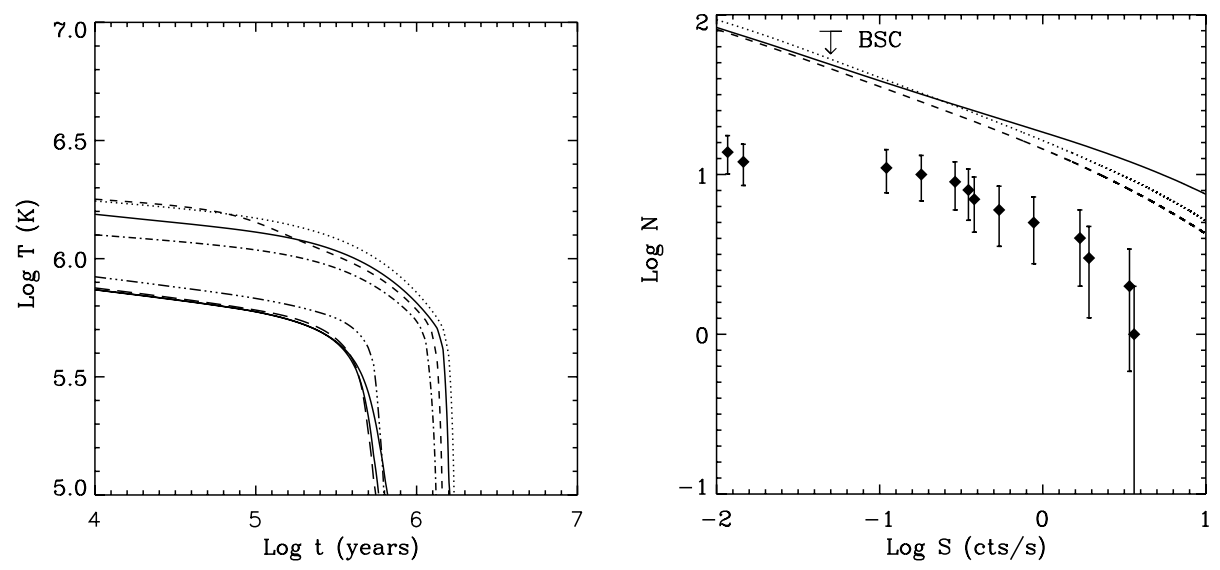

Fig. 4. Same as in Fig. 3 for Model VI. Model IV gives quite similar results since the change of the crust model from C to E does not affect the $\log N-\log S$ distribution.
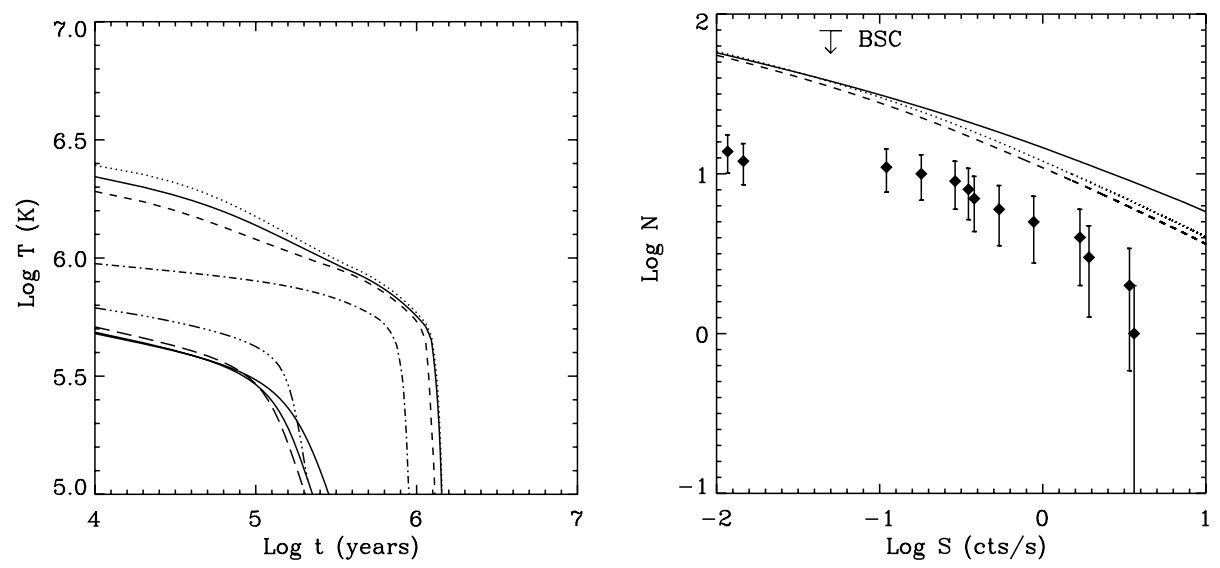

Fig. 5. Same as in Fig. 3 for Model VII. Model XI should produce nearly the same $\log N-\log S$ distribution as it differs only in the type of the crust.

presented by Schwope et al. (1999), on the basis of the ROSAT Bright Sources Catalogue (BSC).

The comparison of the predicted and observed $\log N-$ $\log S$ distributions in Figs. 2-7 indicates that at most three cooling models (model I and possibly models VIII and IX) are in agreement with the data. All the others substantially overpredict the observed number of sources at all fluxes, despite they comply with the T-t test. This latter statement deserves some further comments. BGV did not reject models II-VII on the basis of the fact that the cooling curves cover, for the assumed mass range, the entire region in the T-t plane where the observed sources lie. This approach is certainly sound, and it represents the only possible option to discriminate among different cooling scenarios at the zero level, i.e. without introducing additional information. Clearly, if some assumptions on the NS mass distribution are made, the T-t test can be used to exclude some further models. If the same mass distribution discussed in Sect. 3.2 is applied to BGV sets of cooling curves, one is immediately led to discard models II-V and VII because they predict quite high temperatures for low mass NSs ( $M \lesssim 1.3 M_{\odot}$ ) which, according to our mass spectrum, are very abundant. Although such hot objects would be detectable even to large distances they are not actually observed (this issue is further discussed in G05, see also below). However, care must be taken in using such an argument. Our mass spectrum is meant to be representative of the local population of isolated NSs, and its application to the very biased and limited sample of objects which can be placed on the T-t plots is uncertain. Nevertheless, one can reverse the argument by saying that the two tests, when provided with the same amount of information, yield results which are broadly consistent, as they should. Still, we note that a strict interpretation of the $\log N-\log S$ test results in the exclusion of two further models (VI and VIII) and that even in the most optimistic case model VI is rejected.

The $\log N-\log S$ test is not equally sensitive to changes in three main groups of parameters in Table 1 (presence or absence of the pion condensate, gaps, and type of the crust). Mostly the test reacts to changes in the gap parameters. Oppositely, changes in the type of the crust are not very important as here we consider a sample of relatively old NSs (nevertheless uniform samples of younger NSs can also be studied). Variations in the crust properties are discussed in detail in G05, where the brightness constraint was introduced. G05 shows that models II-V and VII can be ruled when the absence of very bright young NSs is considered as a constraint. The fact that the $\log N-\log S$ test leads to the same conclusion is however a completely independent result, since objects from different age ranges are used in the two approaches. 

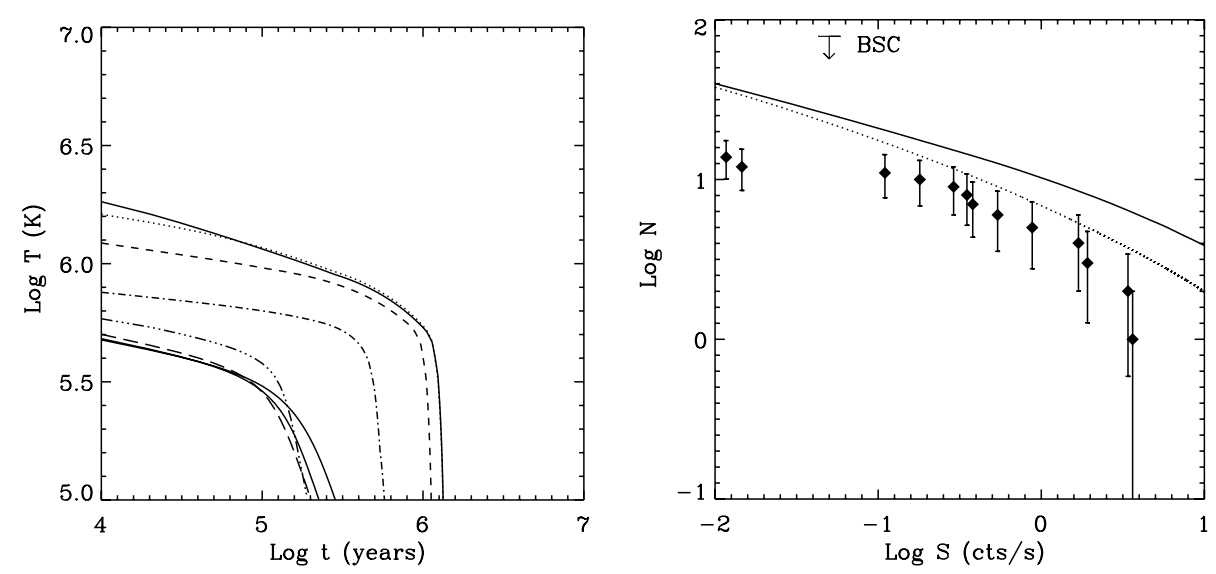

Fig. 6. Same as in Fig. 2 for Model VIII.
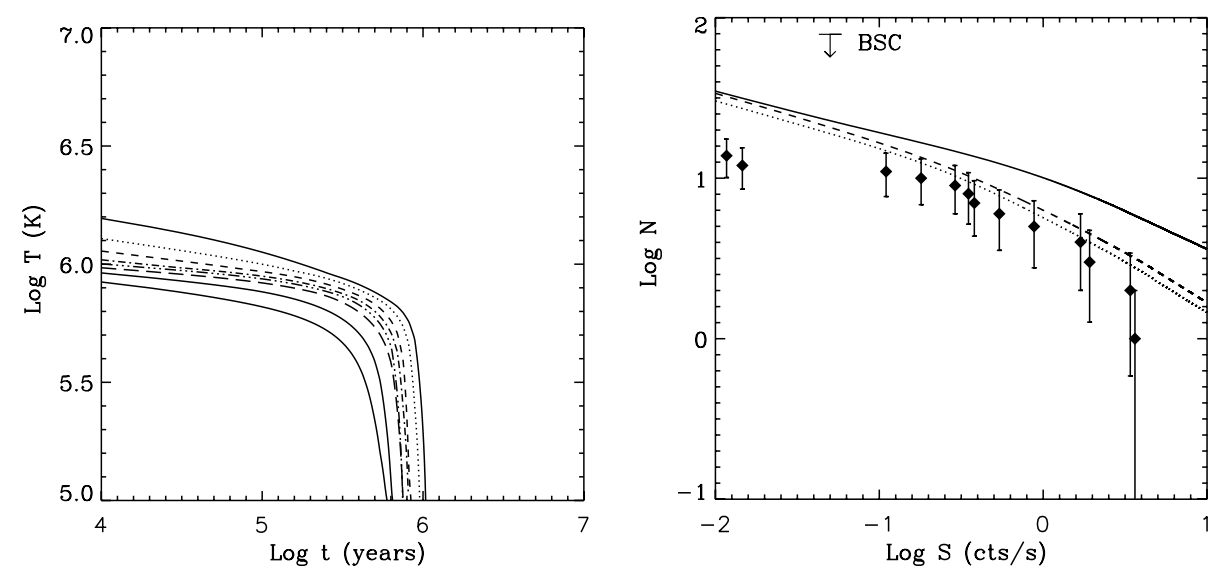

Fig. 7. Same as in Fig. 3 for Model IX.

As mentioned earlier, the $\log N-\log S$ test applied to close-by NSs is not sensitive to changes in the crustal properties. This implies that if a cooling model with a given crust is rejected then its variants with other types of crust (at least from the set considered here) can be ruled out, too. For example, additional models X and XI (see Table 1) do not satisfy the test because their twins (models III and VII, respectively) do not (see Figs. 3 and 5). In G05 it has been shown that it is possible to fullfil the $\mathrm{BC}$ by changing the crust. For example, model III with crust $\mathrm{E}$ can fit the data. However, on the basis of the $\log N-\log S$ test this model is ruled out. This is because the latter test mainly "feels" the changes in gaps. And if they are "wrong" then changes in the crust properties are of no avail.

We want to stress that jumping to the conclusion that the present analysis can provide direct information on the physical state of star interior would be entirely premature. Our adopted scenario contains a number of uncertainties, as discussed in Sects. 2 and 3. Nevertheless, we believe that the case presented here convincingly shows that by combining theoretical cooling curves with populations synthesis calculations one has the potential to discriminate between competing cooling scenarios. In a sense, the main outcome of this investigation is not that models I, and possibly VIII and IX, fit the data while others do not. This may be the result of our starting assumptions. What matters is the fact that, within the same set of assumptions,

different cooling models produce different results when compared with observations.

Current limitations of this approach are related to our present incomplete knowledge of some key issues, chiefly the NS mass spectrum, their surface emission properties and initial spatial distribution. We attempted to account for some of these uncertainties in our model by considering different configurations which should bracket the true behavior. We note that in all the six cases for which no agreement has been found between theoretical and observed distributions, this is largely independent of the assumptions we introduced. Using different values of $R_{\text {belt }}$ or taking variants of the mass spectrum does not help in reconciling predictions with the data. However, we caution that other effects, like those introduced by the proper inclusion of an atmospheric model, may be important. This, and other issues, will be the subject of a future work.

In all the cases we examined (see again Figs. 2-7), with the possible exception of model VIII, the capability of our test to discriminate between different cooling scenarios seems to be quite robust. In this respect, it is interesting to note that the three models (I, VIII and IX) which can reproduce the observed $\log N-\log S$ for our choice of parameters have also been considered by $\mathrm{BGV}$ as the most realistic ones. In fact, among models I-IX which according to BGV are not in contradiction with the temperature-age test, the three 
mentioned above are the theoretically most appealing ones since either the superfluid gaps were calculated with the same nucleon-nucleon interaction which formed the basis for the equation of state and thus the structure of the neutron star configurations (models I and IX) or the gaps were modelled such as to mimic these results (model VIII).

\section{Conclusions}

In this paper we suggest to adopt the $\log N-\log S$ test as an useful addition to the standard T-t test in probing neutron star cooling models. For illustrating the capabilities of the proposed approach, we applied it to nine sets of cooling curves from BGV. Out of sixteen sets described in that paper and two additional ones taken from G05, these eleven produce results which are not in immediate contradiction with the T-t test. The application of the $\log N-\log S$ test rules out at least eight out of eleven investigated cooling models, resulting in just three models which were able to pass both tests. Requiring that the tested cooling models should fulfill in addition the more stringent bightness constraint (G05), there are still six models left out of which only $50 \%$ pass the $\log N-\log S$ test.

One of the most challenging questions for the application of the test suggested in this paper is to use it next for a possible discrimination between purely hadronic compact star cooling scenarios and hybrid ones for stars having a color superconducting quark core (Grigorian et al. 2005) which have already successfully passed the T-t test. Our conclusion is that the $\log N-\log S$ may therefore become a powerful strategy in uncovering the properties of dense nuclear matter under the extreme conditions in neutron star interiors.

Acknowledgements. We thank D. Yakovlev and other members of the St-Petersburg group for many helpful discussions. We are indebted to D. Voskresensky and M. Prokhorov for their constant interest and support. Comments by the referee were very useful and helped to improve the paper. S.P. acknowleges a postdoctoral fellowship from the University of Padova where most of this work has been carried out and a fellowship from the "Dynasty" Foundation. The work of S.P. was partly supported through RFBR grant 03-02-16068. H.G. is grateful to the Department of Physics, University of Padova, for hospitality and acknowledges financial support from the Virtual Institute "Dense hadronic matter and QCD phase transition" of the Helmholtz Association (grant No. VH-VI-041) and from Deutsche Forschungsgemeinschaft (grant No. 436 ARM 17/4/05). The work was partially supported by the Italian Ministry for Education, University and Research under grant PRIN-2002-027245.

\section{References}

Arzoumanian, Z., Chernoff, D. F., \& Cordes, J. M. 2002, ApJ, 568, 289

Blaschke, D., Grigorian, H., \& Voskresensky, D. 2004, A\&A, 424, 979 (BGV)
Bombaci, I., \& Popov, S. B. 2004, A\&A, 424, 627

Bulik, T., Kluzniak, W., \& Zhang, W. 2000, A\&A, 361, 153

Cottam, J., Paerels, F., \& Mendez, M. 2002, Nature, 420, 51

Dall'Osso, S., Israel, G. L., Stella, L., et al. 2003, ApJ, 599, 485

Gotthelf, E. V., \& Vasisht, G. 2000, in Proc. IAU Coll., 177, Pulsar astronomy - 2000 and beyond, ed. M. Kramer, N. Wex, \& N. Wielebinski, ASP Conf. Ser., 202, 699

Geppert, U., Küker, M., \& Page, D. 2004, A\&A, 426, 267

Grenier, I. A. 2000, A\&A, 364, L93

Grigorian, H. 2005 [arXiv: astro-ph/0507052] (G05)

Grigorian, H., Blaschke, D., \& Voskresensky, D. N. 2005, Phys. Rev. C, 71, 045801

Haberl, F. 2004, Adv. Sp. Res., 33, 638

Heger, A., Woosley, S. E., \& Spruit, H. 2005, ApJ, 626, 350

Horvath, J. E. 2004, Int. J. Mod. Phys. D, 13, 1327

Kaminker, A. D., Yakovlev, D. G., \& Gnedin, O. Yu. 2002, A\&A, 383, 1076

Kaspi, V. M., Roberts, M. S. E., \& Harding, A. K. 2004, to appear in Compact Stellar X-ray Sources, ed. W. H. G. Lewin, \& M. van der Klis [arXiv: astro-ph/0402136]

Lattimer, J. M., \& Prakash, M. 2001, ApJ, 550, 426

Neuhäuser, R., \& Trümper, J. 1999, A\&A, 343, 151

Page, D., Lattimer, J. M., Prakash, M., et al. 2004, ApJS, 155, 623

Pavlov, G. G., \& Zavlin, V. E. 2003, in Proc. of the XXI Symposium on Relativistic Astrophysics, ed. R. Bandiera, R. Maiolino, \& F. Mannucci (Singapore: World Scientific Publishing), 319 [arXiv: astro-ph/0305435]

Podsiadlowski, P., Langer, N., Poelarends, A. J. T., et al. 2004, ApJ, 612, 1044

Pöppel, W. 1997, Fund. Cosm. Phys., 18, 1

Popov, S. B., Colpi, M., Prokhorov, M. E., et al. 2000, ApJ, 544, L53

Popov, S. B., et al. 2002, in Proc. of the Third International Sakharov Conference on Physics, ed. A. Semikhatov et al., Scientific World: Moscow, 420 [arXiv: astro-ph/0210688]

Popov, S. B., Colpi, M., Prokhorov, M. E., et al. 2003, A\&A, 406, 111 (Paper I)

Popov, S. B., Turolla, R., Prokhorov, M. E., et al. 2005, Ap\&SS, 299, 117 [arXiv: astro-ph/0305599] (Paper II)

Rutledge, R. E., Fox, D. W., Bogosavljevic, M., et al. 2003, ApJ, 598, 458

Schwope, A. D., Hasinger, G., Schwarz, R., et al. 1999, A\&A, 341, L51

Takatsuka, T., \& Tamagaki, R. 2004, Prog. Theor. Phys., 112, 37

Tammann, G. A., Löffler, W., \& Schröder, A. 1994, ApJS, 92, 487

Thorsett, S. E., \& Chakrabarty, D. 1999, ApJ, 512, 288

Timmes, F. X., Woosley, S. E., \& Weaver, T. A. 1996, ApJ, 457, 834

Torra, J., Fernández, D., \& Figueras, F. 2000, A\&A, 359, 82

Tsuruta, S. 1979, Phys. Rep., 56, 237

Tsuruta, S., Teter, M. A., Takatsuka, T., et al. 2002, ApJ, 571, L143

Woosley, S. E., Heger, A., \& Weaver, T. A. 2002, Rev. Mod. Phys., 74, 1015

Yakovlev, D. G., Levenfish, K. P., \& Shibanov, Yu. A. 1999, Phys. Uspekhi, 42, 737

Yakovlev, D. G., Levenfish, K. P., Potekhin, A. Y., et al. 2004, A\&A, 417, 169 